Received Date : 26-Sep-2016

Revised Date : 17-Nov-2016

Accepted Date : 18-Nov-2016

Article type : Original Paper

\title{
Stent-induced tracheal stenosis can be predicted by IL-8 expression in rabbits
}

Elena Arellano-Orden, $\mathrm{PhD}(1)^{*}$; Carolina Serrano PhD (2)*, Ana Montes-Worboys, $\mathrm{PhD}$

(1); Verónica Sánchez-López, Student (1); Alicia Laborda, PhD (2): Fernando Lostalé, PhD

(2); Celia Lahuerta, $\mathrm{PhD}(2)$; Francisco Rodríguez-Panadero, $\mathrm{PhD}(1,5)$; Miguel Ángel de Gregorio, $\mathrm{PhD}(2,3,4)$.

*These authors contributed equally to this work.

\section{AFFILIATIONS}

1. Unidad Médico-Quirúrgica de Enfermedades Respiratorias, Instituto de Biomedicina de Sevilla (IBiS), Hospital Universitario Virgen del Rocío/Universidad de Sevilla, Seville, Spain.

2. Minimally Invasive Techniques Research Group (GITMI), University of Zaragoza, Zaragoza, Spain.

3. Interventional Radiology, Hospital Clinico Universitario, University of Zaragoza, Zaragoza, Spain.

4 CIBER Bioengineering, Biomaterials and Nanomedicine (BBB) Instituto de Salud Carlos III, Madrid,Spain

This article has been accepted for publication and undergone full peer review but has not been through the copyediting, typesetting, pagination and proofreading process, which may lead to differences between this version and the Version of Record. Please cite this article as doi: 10.1111/eci.12706

This article is protected by copyright. All rights reserved. 
5. CIBER de Enfermedades Respiratorias (CIBERES), Instituto de Salud Carlos III, Madrid, Spain.

\title{
CORRESPONDENCE
}

Francisco Rodriguez-Panadero, M.D., Instituto de Biomedicina de Sevilla (IBiS), Edificio IBiS, Laboratorio 120, Campus H.U. Virgen del Rocio, Avda. Manuel Siurot, s/n, 41013 Sevilla, Spain. Phone: +34 955 923063. E-mail: frodriguezpan@gmail.com

\begin{abstract}
Background: Bare metal stents may cause complications like fibrous encapsulation, granulation and tracheal stenosis. We investigated the behavior of three commercially available stents in vivo (rabbits) and in vitro (co-culture of those stents with epithelial and fibroblast cell lines). Also, we investigated whether development of tracheal stenosis could be predicted by any biological marker.
\end{abstract}

Methods: The tracheae of 30 rabbits were implanted with either nitinol stents, with or without paclitaxel elution, or a cobalt-based stent. An additional ten rabbits underwent mock implantation (controls). Serial peripheral venous blood samples were taken throughout the study, and several cytokines measured. Animals were euthanized on day 90, with immediate tracheal endoscopy and lavage performed, then necropsy.

Results: Rabbits with cobalt-based stent exhibited more inflammation and the highest stenosis incidence, with reduced survival. Both in vivo and in vitro, this stent induced higher IL-8 levels than nitinol stents. Most important, presence of stent-induced tracheal stenosis was closely associated to increase of IL-8 expression in blood just one day after tracheal 
stent implantation: a 1.19 -fold increase vs. baseline had $83 \%$ sensitivity, $83 \%$ specificity, $77 \%$ positive predictive value, $88 \%$ negative predictive value and $83 \%$ accuracy to predict development of stenosis.

Conclusions: The cobalt-based stent had the highest incidence of tracheal inflammation and stenosis. On the other hand, the paclitaxel-eluting nitinol stent did not prevent those complications, and provoked a marked reaction compared with the bare nitinol stent. Early increase of IL-8 expression in blood after stent implantation could predict development of tracheal stenosis in rabbits. Abstract

KEYWORDS: cytokine, rabbits, tracheal stenosis, fibrosis, stents

\section{INTRODUCTION}

Tracheal stents are widely used to treat malignant and benign tracheal stenoses not suitable for surgery $(1,2)$. Silicone stents are frequently used in benign conditions, but require rigid bronchoscopy and general anaesthesia $(3,4)$. Moreover, mucus retention and infections are serious complications with this stent. On the other hand, an increasing array of expandable metallic stents is available, which can be inserted into the airway using a flexible bronchoscope or under fluoroscopy and then expanded to the required diameter. However, most metallic stents are associated with migration, restenosis, granulation tissue, reepithelisation and deep fibrous encapsulation, which make stent removal risky or impossible $(5-10)$.

Drug-eluting stents (DESs) are platforms with deliver anti-proliferative, anti-thrombogenic, anti-inflammatory, or immunosuppressive drugs. Among anti-proliferative drugs, sirolimus (11), everolimus (11-13), and paclitaxel $(14,15)$ have all been widely used. DESs inhibit 
cell proliferation (16-18), and there are theoretical reasons as to why they can be advantageous in the trachea. In this context, research aimed at the prevention of obstruction or fibrotic encapsulation is crucial.

Together with the biological effects associated with the stent composition itself, radial and shear stress forces would have a marked influence on the reaction patterns of the tracheobronchial wall to metallic stents (19). We designed an in vivo and in vitro study, aiming to investigate whether metallic stents (including paclitaxel-eluting stents) induce different tissue reactions. Rabbits were implanted with different stents, which were also cocultured with human respiratory epithelial and fibroblast cell lines. Also, we investigated whether development of tracheal stenosis could be predicted early by any of the markers included in the study.

\section{METHODS}

\section{In vivo study}

Thirty adult female New Zealand rabbits were randomly assigned (ten animals per stent) to receive a paclitaxel-eluting (dose delivery $3 \mu \mathrm{g} / \mathrm{mm}^{2}$ ) nitinol stent (Zilver PTX, Cook Medical, Bjaeverskov, Denmark), a bare nitinol stent (Zilver Flex, Cook Medical, Bjaeverskov, Denmark), or a cobalt-based super alloy stent (Wallstent, Boston Scientific, Natick, MA, USA). The stents were deployed percutaneously under general anaesthesia and fluoroscopic guidance. Computed tomography (CT) tracheal studies were performed under sedation 30 and 90 days after stent deployment as previously published by our Group (20). Parameters assessed in the CT study were: maximum tracheal lumen stenosis in an axial view $(\%)$, stenosis length in a sagittal view $(\mathrm{mm})$, and detection of image compatible with granulomas (present or absent). 
All animals were observed for any sign of respiratory distress or deterioration in general health during the 90-day duration of this experiment. Serial peripheral venous blood samples were taken throughout the study, and animals were euthanized on day 90 by intravenous pentobarbital injection, followed by tracheoscopy, tracheal lavage, and necropsy performed. The following endoscopic aspects were evaluated by two independent reviewers; tracheal stenosis, granulation tissue (polyploid-like lesions), re-epithelisation covering the stent, fibrous reactions (fibrous bands), inflammation, and secretions in the tracheal lumen (Figure $1)$.

The expression of pro-inflammatory (IL-8) and pro-fibrotic cytokines (bFGF, TGF $\beta$ and VEGF) was analysed from peripheral venous blood and tracheal lavage samples using reverse transcription quantikine PCR (RT-qPCR). RNA was isolated using the using High Pure RNA isolation (Roche Mannheim, Germany) cDNA was synthesized using an iScript cDNA synthesis kit (Bio-Rad, Hercules, CA, USA). RT-qPCR was performed using the Stratagene Mx3500 apparatus. $\beta$-actin expression was used as an internal control with which to normalize target gene expression.

Animal care protocols were in compliance with European Communities Council Directive (86/609/EEC), with prior approval from our local Ethics Committee.

\section{In vitro study}

MRC-5 (human lung fibroblast cell line) cells were obtained from the European Collection of Cell Culture (ECACC, Salisbury, UK); Nuli-1 cells (human airway epithelial cell line) were purchased from the American Type Culture Collection (ATCC, Rockville, USA). MRC-5 and Nuli-1 were cultured following the manufacturer's instructions. Cells were 
seeded in $3.3 \mathrm{~cm}^{2}$ wells (Nunc, Roskilde, Denmark) and co-cultured with each stent type for 21 days. Cells cultured without stents served as controls. Each experiment was repeated six times. Culture medium was replaced every three days, with supernatants harvested, aliquoted, and then stored at $-80^{\circ} \mathrm{C}$. Morphological changes in cells were examined by inverted fluorescence microscopy (Zeiss Axiovert 25, Carl Zeiss Co. Oberkochen, Germany). Cell viability determined by trypan blue dye exclusion (Sigma, GmbH, Germany. IL-8, VEGF, bFGF, and TGF- $\beta 1$ levels in culture supernatants were measured by ELISA (R\&D System, Minneapolis, MN, USA), according to the manufacturer's recommendations.

\section{Statistical analysis}

Kaplan-Meier with log-rank (Mantel-Cox) analysis was performed for comparisons of survival. Following normality testing, the student's t-test for independent samples or analysis of variance (ANOVA, with Bonferroni post-hoc test) was used to compare cytokine levels between groups. For non-parametric data, the Mann Whitney U or Kruskal-Wallis tests were applied. For correlations, the Pearson $r$ or Spearman rho coefficients were used, depending on sample characteristics. ROC analysis was used to investigate performance of different parameters in predicting presence of tracheal inflammation or stenosis, and comparisons were made by chi-square test.

Two-tailed $\mathrm{p}$ values $<0.05$ were considered to be significant. Data processing and statistical analyses were performed using SPSS Statistics 20 for Windows.

This article is protected by copyright. All rights reserved. 


\section{RESULTS}

\section{In vivo analyses}

There were no immediate deaths or major complications associated with the procedure; a $100 \%$ technical success rate was achieved for stent implantation.

\section{Tracheoscopy data}

All tracheoscopy findings are presented in Table 1.

Survival was significantly shorter than controls for rabbits implanted with the Wallstent stent (Figure 2A). Short survival was associated to stent-related stenosis (Figure 2B), and those animals had to be euthanized before the experimental deadline.

Stenosis. The highest incidence of stenosis was observed with Wallstent group, and less stenosis was recorded for Zilver Flex (Table 1); nevertheless, the DES Zilver PTX was associated with the most severe stenosis in many cases. Stenosis correlated directly with the presence of inflammation $(\mathrm{r}=0.756, \mathrm{p}<0.001)$, and fibrous reaction $(\mathrm{r}=0.521, \mathrm{p}=0.002)$, and was inversely correlated with survival following stent implantation $(r=-0.404, p=0.02)$. Most important, stenosis was closely associated to increase of IL-8 expression in blood just one day after tracheal stent implantation: a 1.19-fold increase vs. baseline had $83 \%$ sensitivity, $83 \%$ specificity, $77 \%$ positive predictive value, $88 \%$ negative predictive value and $83 \%$ accuracy to predict development of tracheal stenosis.

Granulation tissue. Granulation tissue was observed for all three stent groups, with no significant differences between them. The Zilver PTX was the only stent for which a statistically significant number of granulomas arose in the area underlying the stent, 
compared with more proximal or distal stent locations, which were more commonly seen for the Wallstent group.

Re-epithelisation covering the stent. Re-epithelisation occurred for all animals implanted with the nitinol stents, irrespective of drug delivery; only partial re-epithelisation occurred for the Wallstent group. Of the nitinol stents used, re-epithelisation was most prominent for the Zilver PTX group.

Fibrous encapsulation. Fibrosis was particularly prominent in rabbits with the Zilver PTX stent, compared with other stents $(\mathrm{p}<0.01)$. This adverse effect correlated closely with the extent of re-epithelisation $(\mathrm{r}=0.687, \mathrm{p}<0.001)$, tracheal stenosis $(\mathrm{r}=0.521, \mathrm{p}=0.002)$, and inflammation $(\mathrm{r}=0.48, \mathrm{p}=0.004)$.

Inflammation. Inflammation was evident for all three stent test groups, and was most severe for the Wallstent (85.7\%) versus other groups (62.5\% for Zilver PTX; $22.2 \%$ for Zilver Flex).

\section{Cytokine expression in tracheal lavage and peripheral blood samples}

Pro-inflammatory markers. Rabbits with stents expressed a higher tracheal lavage IL-8 mRNA than controls (Figure 3A). Il-8 mRNA levels in blood are depicted in Figure 3B. When compared with baseline values, the mean IL-8 mRNA-folds at day 1 were 4.49 for Wallstent, 2.82 for Zilver Flex, 2.53 for Zilver PTZ and 0.9 for controls $(p=0.029)$. The single highest tracheal lavage IL-8 mRNA expression (236-fold vs. control) was recorded in a rabbit fitted with a Wallstent that survived only 16 days; this animal had the most severe tracheal inflammation, granulation, and secretions retention.

This article is protected by copyright. All rights reserved. 
Pro-fibrotic gene expression. Tracheal lavage bFGF mRNA levels were lower for all stent groups versus controls $(\mathrm{p}<0.01)$ (Figure 3C), but they were higher than controls in blood. However, they decreased throughout the study (Figure 3D) (see Discussion). Tracheal lavage TGF $\beta$ and VEGF mRNA levels revealed no significant differences between groups (data not shown).

\section{In vitro analyses}

Greater proliferation was noted for the Nuli-1 control cultures versus co-cultures with stents (Figure 4A). Conversely, MRC-5 fibroblasts showed a potent proliferative response around the Wallstent and Zilver Flex stents (Figure 4B). For either cell type, no viable cells could be observed by day 15 co-culture with paclitaxel-delivering stents. When IL-8 levels were normalised to total cell number (IL-8/total cell number ratio), Nuli-1 cells cultured with the Wallstent had the highest IL-8 levels in the first few days of culture (Figure 4C). The normalised data showed more bFGF expression in Nuli-1 stent co-cultures versus controls (Figure 4E).

\section{DISCUSSION}

Stenosis is the hardest problem associated with tracheal stent implantation, and rabbits with the cobalt-based stent had the highest overall incidence in our study. This adverse event was unexpectedly more severe in animals implanted with the paclitaxel-eluting stent. According to serial CT examinations that were published previously by our Group (19), stenosis progressed from day 30 to 90 in nitinol stents (with and without paclitaxel delivery), but not in the cobalt-based alloy stent, which provoked earlier stenosis, subsequently with more early deaths in rabbits. 
Tracheal stenosis was closely associated to increase of IL-8 expression in blood just one day after tracheal stent implantation, with good sensitivity, specificity and accuracy to predict development of stent-induced stenosis in our rabbit study. The question on the mechanisms involved in this systemic IL-8 increase remains open, but it is likely that presence of strong local inflammation in the trachea induces a systemic inflammation also. Other unknown mechanisms, like release of the metallic components, unknown so far, might also be invoked (see below). If extrapolated into humans, our findings would imply that development of stent-induced tracheal stenosis can be predicted when a strong early systemic inflammation is observed after stent placement, and adequate treatment could then be applied to prevent this complication.

On histopathologic examination, the paclitaxel-eluting stent did not behave as expected, and it caused destruction of the epithelium and tracheal structure in seven of the animals $(70 \%)$, with adherence to surrounding tissue. The higher degree of tracheal stenosis observed in the Zilver PTX versus Zilver Flex group (which has the same pattern design and nickel-titanium alloy composition, but without drug delivery) suggests a causal role for paclitaxel. Our findings suggest that paclitaxel at high concentrations is highly injurious to tracheal tissue. While these concentrations might be adequate to prevent neo-intimal hyperplasia following intravascular insertion $(21,22)$, where blood flow clears a portion of the drug delivered, effective clearing is unlikely to occur in the trachea, since the natural mucociliary clearing mechanism can be impaired by stent insertion. Moreover, the lipophilic nature of paclitaxel might enhance cellular uptake, thereby increasing cytotoxicity with subsequent destruction of the normal epithelium, as we described previously (20). 
Re-epithelisation implies a complete covering of the stent that, together with deep fibrous encapsulation, makes stent removal risky or even impossible. The intensity of fibrosis correlated with re-epithelisation, and both were more prominent for the paclitaxel-delivering stent. Fibrosis correlated also with the degree of inflammation and stenosis, which suggests that a complex process involving tissue injury and inadequate repair takes place in rabbits following stent insertion (23), especially with paclitaxel-delivering stents.

Inflammation was more prominent in the Wallstent group, followed by Zilver PTX, and correlated directly with stenosis, granulation tissue, and fibrosis. Moreover, the Wallstent group showed the highest IL-8 production in our in vivo and in vitro study.

As opposed to IL-8, all stent groups had bFGF mRNA downregulated in tracheal lavage samples compared to controls (Figure 3C). However tracheal lavage bFGF correlated positively with survival in our study, which emphasizes the protective role played by this cytokine during tissue injury and repair (24). It is important to note that according to some studies bFGF expression is particularly high in the early phases of granulation tissue formation, and then gradually decreases in the mature phase of post-intubation tracheal stenosis (25), which is compatible with our findings in blood samples in our study (Figure 3D).

As with other lesions, granulation tissue formation was higher for the Wallstent group, especially in areas proximal or distal to the stents. Since all stents had the same size $(8 \times 40$ $\mathrm{mm}$ ), the differences found in biologic reactions to the stents were probably related to their different geometric structures and/or composition. The Wallstent was made of stainless steel filaments in the past and, more recently, an alloy containing cobalt, chromium, nickel, 
molybdenum and manganese, amongst other elements. The filaments are organized in a crisscross pattern and have an open strut structure at both the proximal and distal ends, which may be responsible for the tension-associated lesions observed at those locations. On the other hand, Zilver Flex and PTX are made of a nickel-titanium (nitinol) super alloy with memory shape and a zigzag structure, with less tension exerted on the tracheal wall. By building a rabbit trachea numerical model, our Group was able to analyse the behaviour of the trachea before and after deployment of Zilver Flex and Wallstent stents (the same that were used for this present study) and thus showed that tensile stent-associated forces may lead to a greater tracheal epithelium response within the stented region (26).

Role of the in vitro study. Our in vivo rabbit study involved both biological effects derived from the composition of the stent itself and mechanical forces exerted by the stent on the surrounding tissue, and distinguishing between the two types of influence is not a trivial exercise. Aiming to overcome this problem, we combined in vivo experiments with coincubation of the same metallic stents with epithelial and fibroblast cell lines in vitro.

All stents induced significantly higher IL-8 levels in culture compared to controls, with the Wallstent group generating the most IL-8, which agreed with our in vivo data suggesting that composition of the stent itself was the cause of the systemic pro-inflammatory response observed in rabbits. We speculate that this systemic inflammation might be related to the release of metallic elements from the stent alloys, and also suspect that the results obtained in our in vitro study could be caused, at least in part, by that release (lixiviation) (27). These aspects deserve further investigation in the future.

This article is protected by copyright. All rights reserved. 


\section{Limitations of the study}

The high rate of complications found with the paclitaxel eluting stent was probably related to the high and fast delivery of this drug to the surrounding tracheal tissue. Establishing the correct dosage for specific tissue targets is therefore important (28). A previous in vitro study from our group suggested that cell viability could be better preserved using a paclitaxel-delivering stent with a dose delivery of $1 \mu \mathrm{g} / \mathrm{mm}^{2}$, rather than the $3 \mu \mathrm{g} / \mathrm{mm}^{2}$ currently delivered by the Zilver PTX stent (29), then supporting the concept that too high doses of paclitaxel have played a critical role in the destructive patterns that we observed with this stent implanted on rabbits. Using stents in conjunction with other anti-proliferative drugs $(29,30)$ might represent plausible alternatives, and drug-eluting bio absorbable stents might also play a role in the future (31).

Although performance of the in vivo experiments with stents inserted in a normal (nonstenotic) rabbit trachea is another limitation in our study, our approach provided a uniform baseline status for all animals, then allowing investigation of damage caused by the stents themselves. Our study was performed in small animals that required narrow vascular stent insertion, and it could be argued that our results do not readily extrapolate to humans. We would agree with this, but we also believe that the combination of our in vitro and in vivo approaches provides relevant insights into the pathophysiological mechanisms that are involved in stent-associated tissue damage.

\section{CONCLUSIONS}

Metallic stents, with and without paclitaxel delivery, induced a marked pro-inflammatory response in the rabbit trachea. The paclitaxel-eluting nitinol stent provoked a stronger reaction than bare nitinol, and the cobalt-based stent had the highest pro-inflammatory 
response. Most important, tracheal stenosis was closely associated to increase of IL-8 expression in blood just one day after tracheal stent implantation. If extrapolated into humans, our findings would imply that development of stent-induced tracheal stenosis can be predicted in advance when a strong systemic inflammation is observed early after stent placement, and adequate treatment could then be applied to prevent this complication.

\section{ACKNOWLEDGEMENTS}

None of the authors have any conflict of interest to declare. All authors have read the Translational Research's policy on conflicts of interest. All authors have read the journal's authorship agreement, and the article has been reviewed by and approved by all named authors.

This study was supported by Grants FIS 08/0096 and FIS 08/1424 from the Instituto de Salud Carlos III, Madrid, Spain, and by Grant Neumosur 4/2007.

AUTHOR CONTRIBUTIONS: FRP and MADG designed the study. CS, AL and MADG performed the in vivo experiments on rabbits. CS and FRP rated independently the findings at tracheoscopy in rabbits. EAO, VSL, CL and AMW performed the in vitro experiments with respiratory cell lines. FL and CS performed autopsies and histology analysis. FRP, MADG, CS, EAO and FL contributed to the data analysis. EAO, CS, AMW, VSL, AL, FL, FRP and MADG participated in the writing of the manuscript, revision of the drafts and approval of the final version submitted. FRP and MADG are guarantors of the contents of the manuscript.

This article is protected by copyright. All rights reserved. 


\section{References}

1. Ernst A, Feller-Kopman D, Becker HD, Mehta AC. Central airway obstruction. American journal of respiratory and critical care medicine. 2004;169(12):1278-97.

2. Bolliger CT, Mathur PN, Beamis JF, Becker HD, Cavaliere S, Colt H, et al. ERS/ATS statement on interventional pulmonology. European Respiratory Society/American Thoracic Society. The European respiratory journal. 2002;19(2):356-73.

3. Noppen M, Meysman M, Claes I, D'Haese J, Vincken W. Screw-thread vs Dumon endoprosthesis in the management of tracheal stenosis. Chest. 1999;115(2):532-5.

4. Bolliger CT, Wyser C, Wu X, Hauser R, Studer W, Dalquen P, et al. Evaluation of a new selfexpandable silicone stent in an experimental tracheal stenosis. Chest. 1999;115(2):496-501.

5. Saad CP, Murthy S, Krizmanich G, Mehta AC. Self-expandable metallic airway stents and flexible bronchoscopy: long-term outcomes analysis. Chest. 2003;124(5):1993-9.

6. Kim H. Stenting therapy for stenosing airway disease. Respirology. 1998;3(4):221-8.

7. Lunn W, Feller-Kopman D, Wahidi M, Ashiku S, Thurer R, Ernst A. Endoscopic removal of metallic airway stents. Chest. 2005;127(6):2106-12.

8. Rampey AM, Silvestri GA, Gillespie MB. Combined endoscopic and open approach to the removal of expandable metallic tracheal stents. Archives of otolaryngology--head \& neck surgery. 2007;133(1):37-41.

9. Alazemi S, Lunn W, Majid A, Berkowitz D, Michaud G, Feller-Kopman D, et al. Outcomes, health-care resources use, and costs of endoscopic removal of metallic airway stents. Chest. 2010;138(2):350-6.

10. Lund ME, Force S. Airway stenting for patients with benign airway disease and the Food and Drug Administration advisory: a call for restraint. Chest. 2007;132(4):1107-8.

11. Weinberg SL. Toward defining the role of drug-eluting stents. The American heart hospital journal. 2007;5(3):133-4.

12. Zohlnhofer D, Klein CA, Richter T, Brandl R, Murr A, Nuhrenberg T, et al. Gene expression profiling of human stent-induced neointima by cDNA array analysis of microscopic specimens retrieved by helix cutter atherectomy: Detection of FK506-binding protein 12 upregulation. Circulation. 2001;103(10):1396-402.

13. Roque M, Cordon-Cardo C, Fuster V, Reis ED, Drobnjak M, Badimon JJ. Modulation of apoptosis, proliferation, and p27 expression in a porcine coronary angioplasty model. Atherosclerosis. 2000;153(2):315-22.

14. Choritz L, Grub J, Wegner M, Pfeiffer N, Thieme H. Paclitaxel inhibits growth, migration and collagen production of human Tenon's fibroblasts--potential use in drug-eluting glaucoma drainage devices. Graefe's archive for clinical and experimental ophthalmology = Albrecht von Graefes Archiv fur klinische und experimentelle Ophthalmologie. 2010;248(2):197-206.

15. Stone GW, Ellis SG, Cox DA, Hermiller J, O'Shaughnessy C, Mann JT, et al. A polymer-based, paclitaxel-eluting stent in patients with coronary artery disease. The New England journal of medicine. 2004;350(3):221-31.

16. Deconinck E, Sohier J, De Scheerder I, Van den Mooter G. Pharmaceutical aspects of drug eluting stents. Journal of pharmaceutical sciences. 2008;97(12):5047-60.

17. Sheiban I, Villata G, Bollati M, Sillano D, Lotrionte M, Biondi-Zoccai G. Next-generation drugeluting stents in coronary artery disease: focus on everolimus-eluting stent (Xience V). Vascular health and risk management. 2008;4(1):31-8.

18. Ong AT, Serruys PW. Technology Insight: an overview of research in drug-eluting stents. Nature clinical practice Cardiovascular medicine. 2005;2(12):647-58.

19. Grewe PH, Muller KM, Lindstaedt M, Germing A, Muller A, Mugge A, et al. Reaction patterns of the tracheobronchial wall to implanted noncovered metal stents. Chest. 2005;128(2):986-90.

This article is protected by copyright. All rights reserved. 
20. Serrano C, Lostale F, Rodriguez-Panadero F, Blas I, Laborda A, de Gregorio MA. Tracheal SelfExpandable Metallic Stents: A Comparative Study of Three Different Stents in a Rabbit Model. Archivos de bronconeumologia. 2015.

21. Heldman AW, Cheng L, Jenkins GM, Heller PF, Kim DW, Ware M, Jr., et al. Paclitaxel stent coating inhibits neointimal hyperplasia at 4 weeks in a porcine model of coronary restenosis. Circulation. 2001;103(18):2289-95.

22. Gershlick A, De Scheerder I, Chevalier B, Stephens-Lloyd A, Camenzind E, Vrints C, et al. Inhibition of restenosis with a paclitaxel-eluting, polymer-free coronary stent: the European evaLUation of pacliTaxel Eluting Stent (ELUTES) trial. Circulation. 2004;109(4):487-93.

23. Morishima $Y$, Nomura A, Uchida $Y$, Noguchi $Y$, Sakamoto $T$, Ishii $Y$, et al. Triggering the induction of myofibroblast and fibrogenesis by airway epithelial shedding. American journal of respiratory cell and molecular biology. 2001;24(1):1-11.

24. Nakanishi R, Hashimoto M, Yasumoto K. Improved airway healing using basic fibroblast growth factor in a canine tracheal autotransplantation model. Annals of surgery. 1998;227(3):44654.

25. Cai Z, Li H, Zhang H, Han S, An R, Yan X. Novel insights into the role of hypoxiainducible factor1 in the pathogenesis of human postintubation tracheal stenosis. Molecular medicine reports. 2013;8(3):903-8.

26. Chaure J, Serrano C, Fernandez-Parra R, Pena E, Lostale F, Gregorio MA, et al. On Studying the Interaction Between Different Stent Models and Rabbit Tracheal Tissue: Numerical, Endoscopic and Histological Comparison. Annals of biomedical engineering. 2015.

27. Cadosch D, Chan E, Gautschi OP, Filgueira L. Metal is not inert: role of metal ions released by biocorrosion in aseptic loosening--current concepts. Journal of biomedical materials research Part A. 2009;91(4):1252-62.

28. Lee SS, Shin JH, Han JM, Cho CH, Kim MH, Lee SK, et al. Histologic influence of paclitaxeleluting covered metallic stents in a canine biliary model. Gastrointestinal endoscopy. 2009;69(6):1140-7.

29. Arellano Orden E MWA, Sáenz Coronilla FJ, Serrano Casorran C, Rodríguez Panadero F. Interacciones entre stents metálicos desnudos y liberadores de droga con fibroblastos respiratorios co-cultivados in vitro. Rev Esp Patol Torac. 2013;25:235-47.

\section{Legends for figures:}

Figure 1. Evaluation of endoscopic aspects. Panel A, tracheal stenosis: rated from 0

(absent) to 2 (severe); panel B, granulation tissue (polyploid-like lesions): from 0 (absent) to 1 (present); panel C, re-epithelisation over the stent surface: rated from 0 (absent) to 3 (complete with stent covered by thick tissue); panel D, fibrous reaction (fibrous bands): from 0 (absent) to 2 (severe); panel E, inflammatory aspect of the tracheal surface: from 0 (absent) to 2 (severe); panel F, secretions in the tracheal lumen: from 0 (absent) to 2 (severe). 
Figure 2. Survival of rabbits. Panel A. Survival following stent implantation. KaplanMeier analysis. Panel 2B: Survival of rabbits with/without stent-induced tracheal stenosis.

Figure 3. Gene expression following stent implantation. Panel A: Tracheal lavage IL-8 expression (fold versus control). Panel B: IL-8 expression in serial blood samples. Panel C: Tracheal lavage bFGF expression (fold versus control). Panel D: bFGF expression in serial blood samples. $(*) \mathrm{p}<0.05$ and $(* *) \mathrm{p}<0.001$.

Figure 4. Results of the "in vitro" study. Morphologic changes for 21 day co-cultures of Nuli-1 (Panel A) and MRC-5 (Panel B) cells with each stent (10X). IL-8 levels $((\mathrm{pg} / \mathrm{ml}) /$ total cells in supernatants of Nuli-1 (Panel C) and MRC-5 (Panel D). bFGF levels $((\mathrm{pg} / \mathrm{mL}) /$ total cells) in cell culture of Nuli-1 (Panel E) and MRC-5 (Panel F). WS, Wallstent chrome-cobalt super alloy; ZF, Zilver Flex, nickel-titanium (nitinol) alloy; PTX, Zilver PTX (nitinol covered with paclitaxel, $3 \mu \mathrm{g} / \mathrm{mm}^{2}$ ); control, cell culture with no stent. 
Table 1. Findings at tracheoscopy for the Wallstent, Zilver Flex, Zilver PTX and control groups.

\begin{tabular}{|c|c|c|c|c|}
\hline $\begin{array}{c}\text { Findings at } \\
\text { tracheoscopy }\end{array}$ & Wallstent & Zilver Flex & Zilver PTX & Control \\
\hline Stenosis & $1 \pm 0.1^{* *}$ & $0.2 \pm 0.1$ & $0.7 \pm 0.3^{*}$ & 0 \\
\hline Granulation & $0.7 \pm 0.2^{*}$ & $0,6 \pm 0.2^{*}$ & $0.6 \pm 0.2^{*}$ & 0 \\
\hline tissue & & & & 0 \\
\hline Re-epithelisation & $1 \pm 0.1^{* *}$ & $2 \pm 0.1^{* *}$ & $2.5 \pm 0.2^{* *}$ & 0 \\
\hline Fibrous bands & & & & 0 \\
\hline Inflammation & $1.9 \pm 0.1^{* *}$ & $1.2 \pm 0.1^{* *}$ & $1.6 \pm 0.2^{* *}$ & \\
\hline $\begin{array}{c}\text { Survival (median } \\
\text { and outliers) }\end{array}$ & $46(8-90)^{* *}$ & $90(5-90)$ & $84(1-90)$ & $00(73-90)$ \\
\hline Secretions & $1.6 \pm 0.2^{* *}$ & $0.9 \pm 0.2^{* *}$ & $0.6 \pm 0.3$ & \\
\hline & & & & \\
\hline
\end{tabular}

Tracheal stenosis: rated from 0 (absent) to 2 (severe); granulation tissue (polyploid like lesions): from 0 (absent) to 1 (present); re-epithelisation over the stent surface: rated from 0 (absent) to 3 (complete with stent covered by thick tissue); fibrous reaction (fibrous bands): from 0 (absent) to 2 (severe); inflammatory aspect of the tracheal surface: from 0 (absent) to 2 (severe); secretions in the tracheal lumen: from 0 (absent) to 2 (severe). Scores are 
presented as mean \pm SEM, unless otherwise stated. (*) Statistical differences $(\mathrm{p}<0.05) ;(* *)$

Statistical differences $(\mathrm{p}<0.01)$.

Figure 1. Evaluation of endoscopic aspects. Panel A, tracheal stenosis: rated from 0

(absent) to 2 (severe); panel B, granulation tissue (polyploid-like lesions): from 0 (absent) to

1 (present); panel C, re-epithelisation over the stent surface: rated from 0 (absent) to 3

(complete with stent covered by thick tissue); panel D, fibrous reaction (fibrous bands):

from 0 (absent) to 2 (severe); panel E, inflammatory aspect of the tracheal surface: from 0

(absent) to 2 (severe); panel F, secretions in the tracheal lumen: from 0 (absent) to 2

(severe).

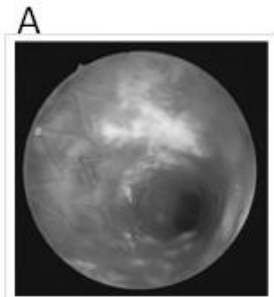

Absent

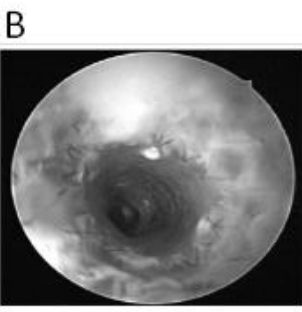

No granuloma

C

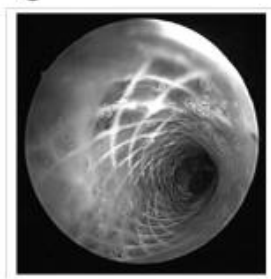

Partial re-epithelation (rating 1)

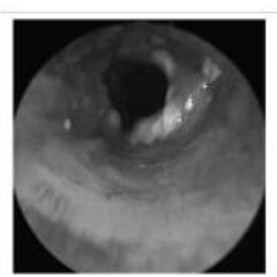

Medium

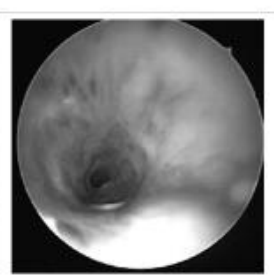

Severe

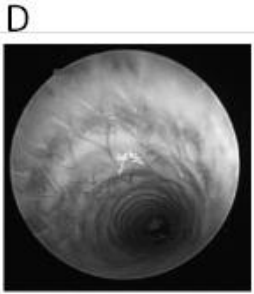

No fibrous reaction E

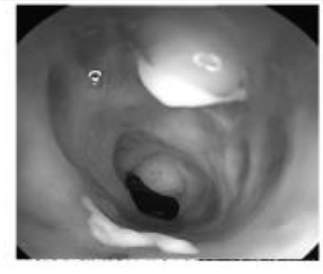

Granuloma (+ Fibrous reaction)

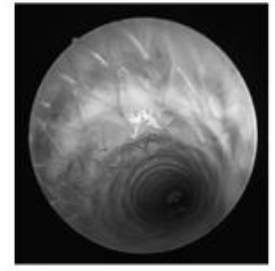

Complete re-epitheliation Stent fully covered (rating 2) (rating 3)

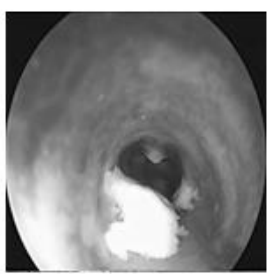

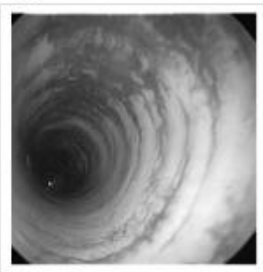
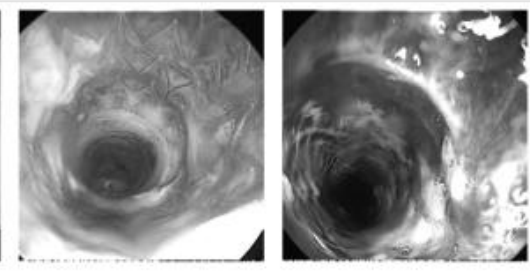

No inflammation F

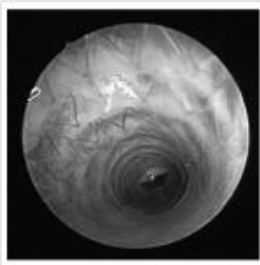

Absence of secretions

Moderate inflammation Severe inflammation

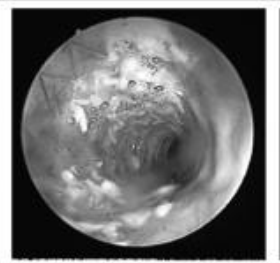

Moderate secretions
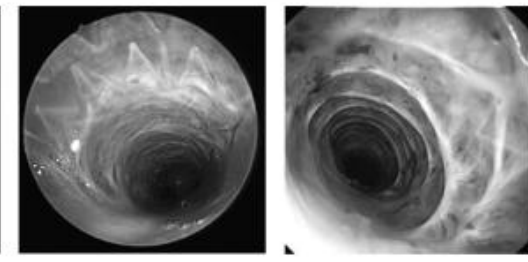

Moderate fibrous reaction Severe fibrous reaction

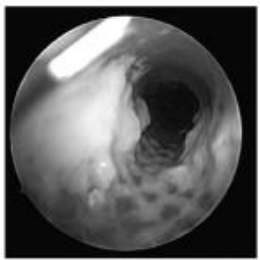

Severe (rating 2)

This article is protected by copyright. All rights reserved. 
Figure 2. Survival of rabbits. Panel A. Survival following stent implantation. KaplanMeier analysis. Panel 2B: Survival of rabbits with/without stent-induced tracheal stenosis.

A

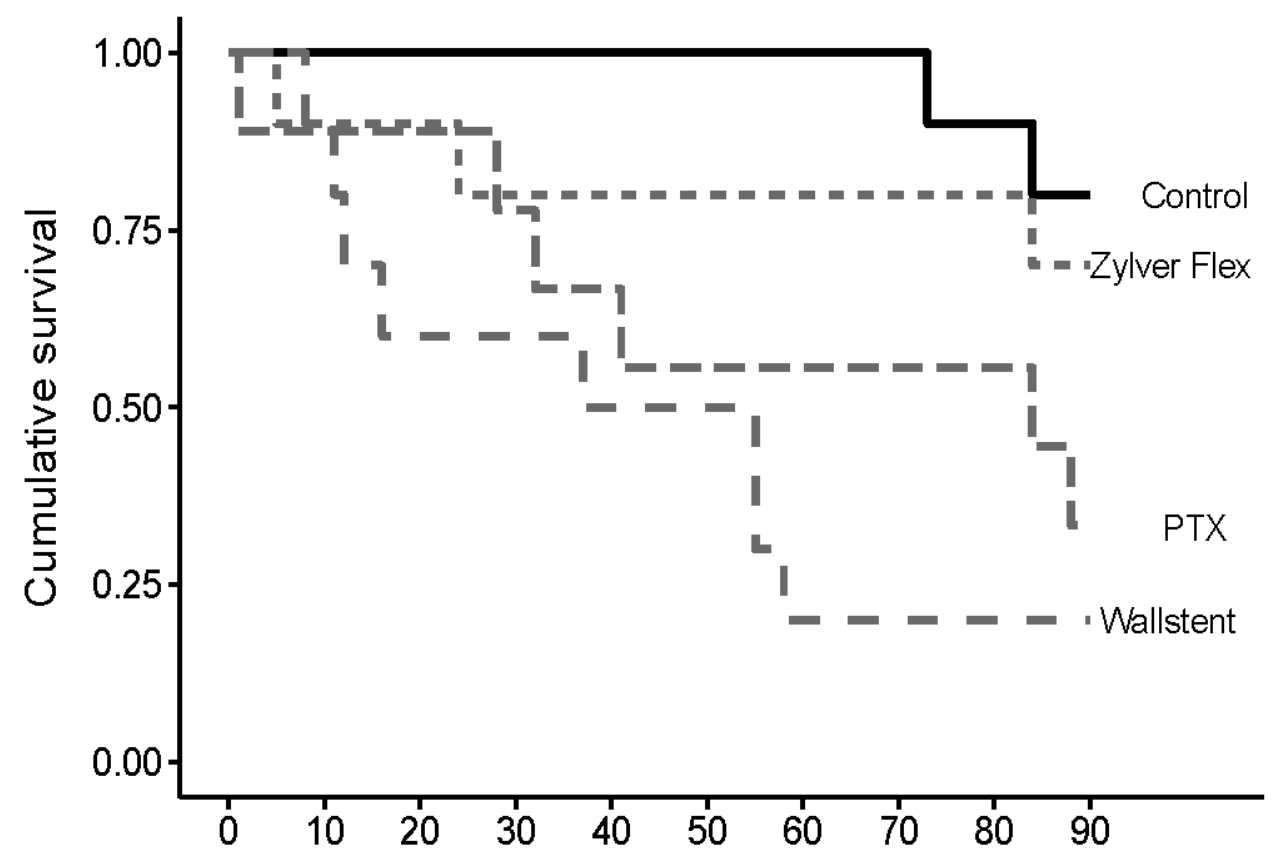

B

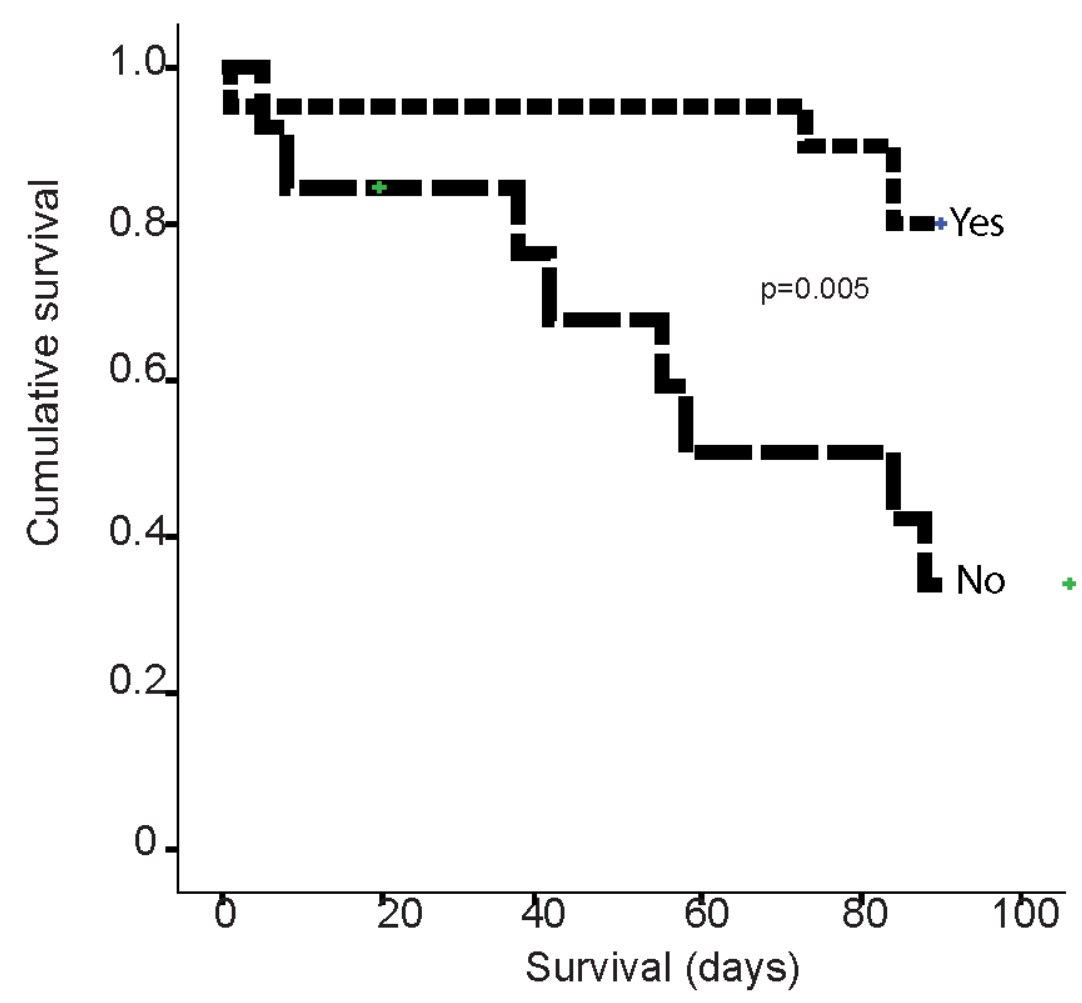

This article is protected by copyright. All rights reserved. 
Figure 3. Gene expression following stent implantation. Panel A: Tracheal lavage IL-8 expression (fold versus control). Panel B: IL-8 expression in serial blood samples. Panel C: Tracheal lavage bFGF expression (fold versus control). Panel D: bFGF expression in serial blood samples. $\left(^{*}\right) \mathrm{p}<0.05$ and $(* *) \mathrm{p}<0.001$.

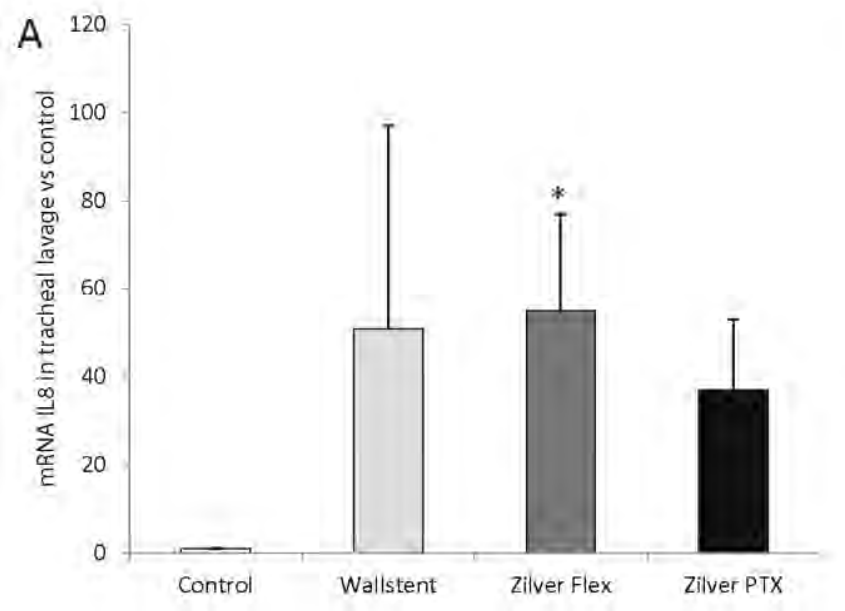

B
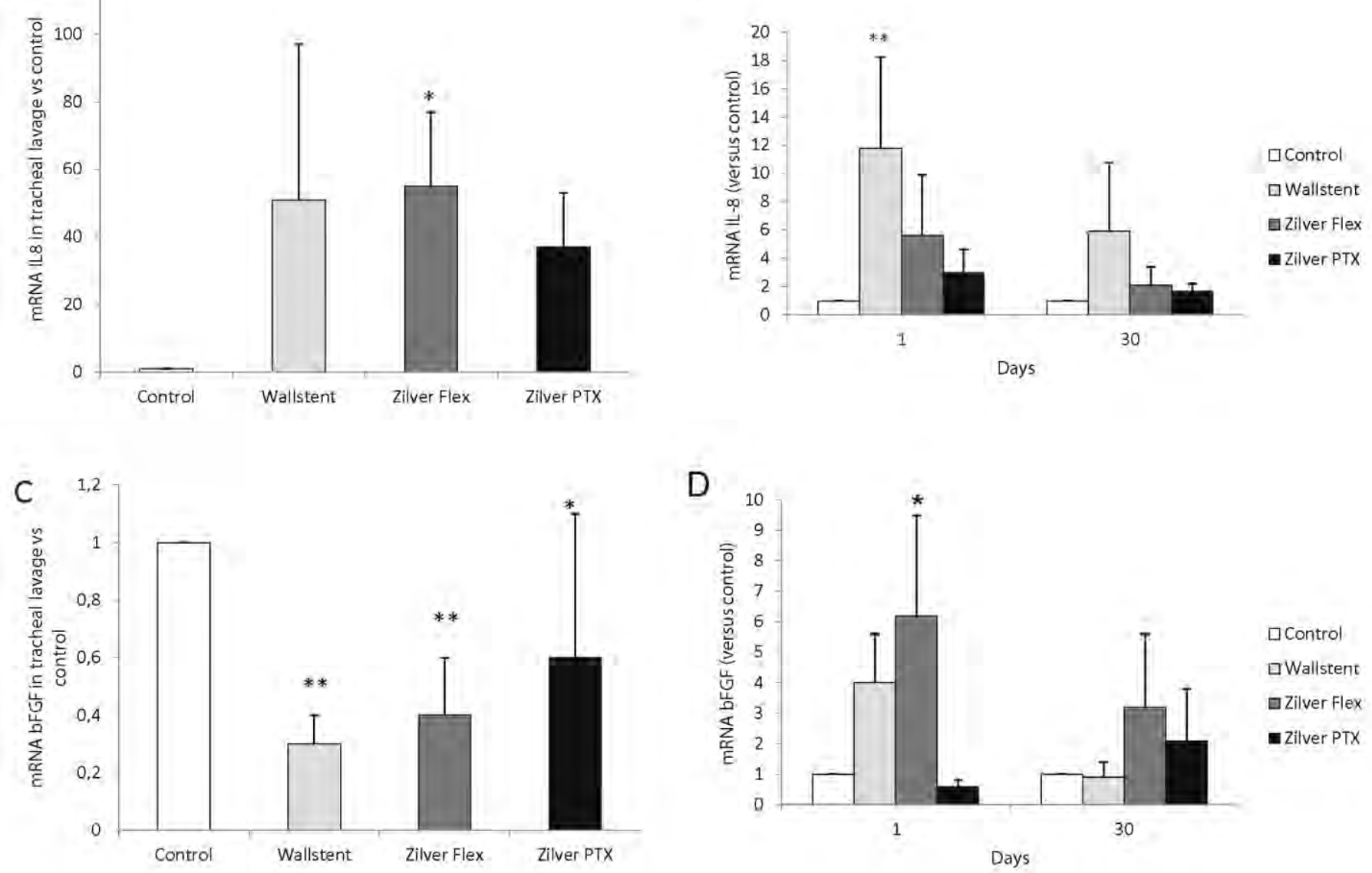

This article is protected by copyright. All rights reserved. 
Figure 4. Results of the "in vitro" study. Morphologic changes for 21 day co-cultures of Nuli-1 (Panel A) and MRC-5 (Panel B) cells with each stent (10X). IL-8 levels $((\mathrm{pg} / \mathrm{ml}) /$ total cells in supernatants of Nuli-1 (Panel C) and MRC-5 (Panel D). bFGF levels $((\mathrm{pg} / \mathrm{mL}) /$ total cells $)$ in cell culture of Nuli-1 (Panel E) and MRC-5 (Panel F). WS, Wallstent chrome-cobalt super alloy; ZF, Zilver Flex, nickel-titanium (nitinol) alloy; PTX, Zilver PTX (nitinol covered with paclitaxel, $3 \mu \mathrm{g} / \mathrm{mm}^{2}$ ); control, cell culture with no stent.

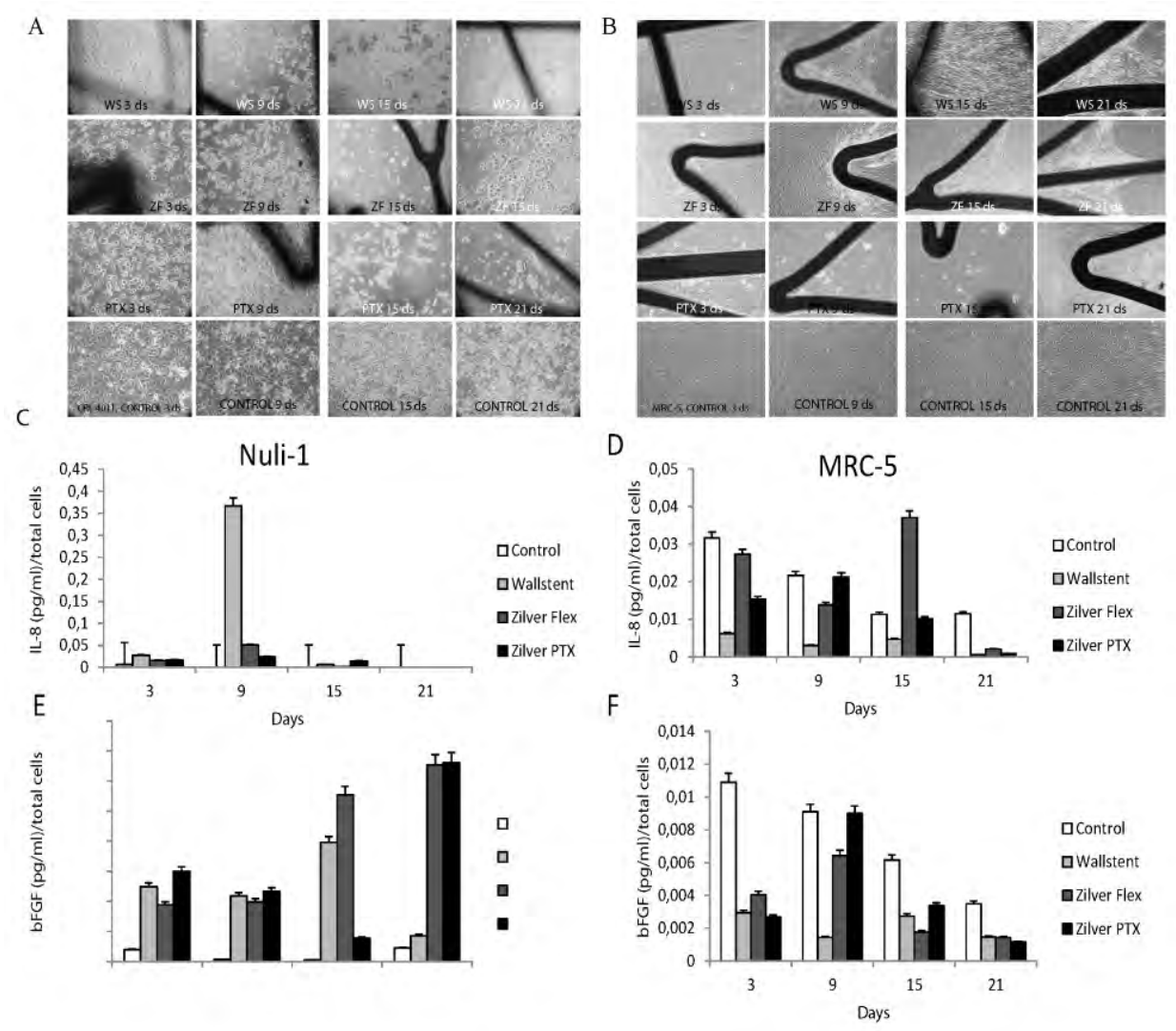

